\title{
Vehicles Counting from Video Stream for Automatic Traffic flow Analysis Systems
}

\author{
Nur Adilah Mohd ${ }^{1}$, Salama A. Mostafa ${ }^{1}$, Aida Mustapha ${ }^{1}$, Azizul Azhar Ramli ${ }^{1}$, Mazin Abed Mohammed ${ }^{2}$ \\ Nallapaneni Manoj Kumar ${ }^{3}$ \\ ${ }^{1}$ Faculty of Computer Science and Information Technology, \\ UniversitiTun Hussein Onn Malaysia, Parit Raja, 86400 Johor, Malaysia \\ ${ }^{2}$ College of Computer Science and Information Technology, University of Anbar, 11 Ramadi, 55 Anbar, Iraq \\ ${ }^{3}$ School of Energy and Environment, City University of Hong Kong, Kowloon, Hong Kong \\ nuradilah.mohd97@gmail.com, \{salama, aidam, azizulr\}@uthm.edu.my, \\ mazinalshujeary@uoanbar.edu.iq and nallapanenichow@gmail.com
}

\begin{abstract}
Recently, video-based and real-time vehicle counting become a popular approach for Traffic Flow Analysis (TFA). One of the main objectives of this analysis is to solve the problems that cause traffic congestion including identifying peak hours. Subsequently, this paper proposes an Automatic Video-based Vehicles Counting (AVVC) model for accurate vehicle counting from video streams.The AVCV model includesactive contour which is used to detect whether the object is a vehicle or not, Gaussian distribution which is used for background subtraction and Bilateral Filter which is used for removing shadow and also for smoothing the image. Besides, Kalman Filter is used to reduce the noise in the imagesand Histogram of Oriented Gradient (HOG) and Hough Transform algorithms are used to improve the accuracy of the counting by enabling the model to distinguish between two overlapped objects of vehicles. Hence, our contribution is a strong segmentation algorithm that detects foreground pixels of objects corresponding to moving vehicles.The model is tested and evaluated in terms of counting accuracy and precision using standard dataset video records of three different locations. The AVVC model achieves vehicles counting accuracy of 95.14and precision of $92.81 \%$ on average.
\end{abstract}

Key words: Traffic Flow Analysis (TFA), vehicles counting, Active Contour, Gaussian distribution, Kalman Filter.

\section{INTRODUCTION}

Intelligent Traffic Flow Analysis (TFA) systems include sensing and data processing techniques that attempt to improve the traditional traffic systems [1]. They integrate additional hardware of sensors, digital traffic signs and cameras and apply advanced techniques to process the data provided by the hardware to intelligently improve vehicles and traffic flow [2]. For example, efficiently adjusting the lights phasing and timing of a traffic light system in such a way that improves the traffic flow and reduce the delay [3].

In avideo-based vehicle counting, the TFA system makes use of videos stream that contains a sequence of images [4]. This is essential when digital image processing is the only practical technology for counting, feature extraction, pattern recognition, projection and multi-scale signal analysis of the system which can know the vehicle counting, the system also needs to identify the exact location to be more accurate in counting vehicles [5]. Basically, the video is divided into frames, the frames are further transformed into colored or gray level frames and the frames are given to the system as inputs. Then the system applies different types of algorithms for preprocessing, object detection, identification and tracking to perform vehicle counting [6]. The tracking is focusing on a particular region of animage which is considered to be important for the collection of data called as Region of Interest (ROI)and after the system collectsthe datait will ensure not to reconsider processing/counting the same object again.Vehicle tracking is an entire measurement process in many defined frames for the same object location [7].

The review of equivalent existing systems supports additional information on designing the proposed solution model. It shows the combinations of the methods and algorithms of vehicle detection and counting from traffic surveillance video sequences. It identifies five main processing steps in vehicles counting which are preprocessing, segmentation, object detection, vehicle counting, and vehicle tracking. Some similar works in vehicle counting include the recent work of Chhadikar et al. [4]. They use a Haar-Cascade method to detect objects. The method includes a haar-like function to identify a rectangular element in a particular image. The Haar-like function offers high rate calculations according to the number of pixels in the right-hand side of the image and not according to each pixel. Then the counting of vehiclesis performed. The method comprises a tracking mechanism that comparesidentified objects with a list of tracked items for each point in the ROI to avoid multiple counting [2]. 
In Crouzil et al. [2] work, the processing of vehicles countingis performed as shadow removal, background removal, motion detection, countingand tracking the shadow detection entails that a pixel in a light area is darker than the same pixel in a shadowed zone. Kalman filter is adapted for movements rectilinear and smooth.Adi et.al., (2018) propose a lane masking, counting and detection method. The method distinguishes sections of the image that are included in the computation for the lane masking process. Some important parts of the imageare multiplied by 1 and other not important partsare multiplied by 0 to represent the ROI. This results in the pictures having only the required machine bits. This technique can reduce calculation time and background noise.

In Soleh et al.[6], Theyuse segmentation method for the object of the vehicles to connect unbounded parts of the objects or to close the holesof the dilation. The detection of vehicle objects is performed by determining the ROI through the Blob detection segmentation. The moment function is used to define a centroid value for each vehicle feature. The identification of the vehicle is performed by processing phase using Gaussian Mixture Distribution and Post-Processing phases using Optical Flow Density and Farnebackalgorithms. The Optical flow density is a motion path that reveals the presence of an object in two frames induced by the motion of the object or camera.They useHungarian Kalman Filter to distinguishing between different objects.

This project is dedicated to the presentation of a vision-based system for road vehicle counting due to the user always faced traffic flow congestion, especially during peak hour. An Automatic Video-based Vehicles Counting (AVVC) model is proposed for accurate vehicle counting from a video stream. The AVVC model uses Gaussian distribution and Kalman Filter for image processing, and optical flow for tracking and active contour for detection. Besides, we use Histogram of Oriented Gradient (HOG) and Hough Transform for the overlapped image and object orientation. The model is able to achieve very good counting accuracy with different traffic conditions.Our main contribution represented by constructing a strong segmentation algorithm that detects foreground pixels of objects corresponding to moving vehicles.

\section{MATERIALS AND METHODS}

This project is dedicated to the presentation of a vision-based system for road vehicle counting at Johor (highway, major-road, sub-road) area due to the user always faced the traffic flow congestion, especially during peak hours. The development methodology of this work ensures that all the contents and supporting data are reviewed and verifiable. This methodologypresent requirements that provide an overview of the project life-cycle. Fig 1 shows the main processes of the research methodology.

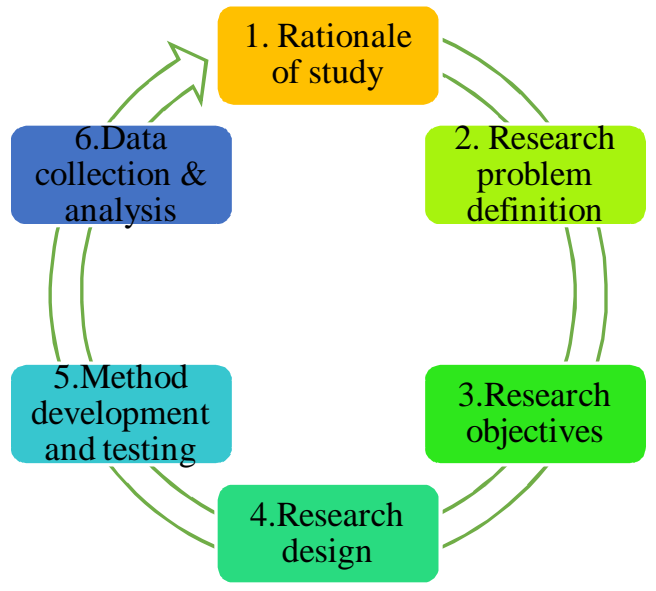

Figure 1: The processes of the Research Methodology.

In this project, the problem is the accuracy of the vehicle countingbased on video streaming approach. Subsequently, we attempt to build a model that improves the accuracy of vehicle counting [9] [10] [11].The aim of the model is to be deployed in automatic TFA systems. The research outcomes the development of the AVVC model.Testing is properly performed to detect errors or other problems before making the developed model available for the validity test. The tested parameters related to the vehicles counting are as follow:

- Number of Vehicles each lane

- Total number of vehicle

- Critical lane

- Peak traffic time

\subsection{Dataset}

The Sochoret al. [12]BrnoCompSpeeddataset of this project is regular video-stream recordscaptured at six different locations. The BrnoCompSpeed dataset includesa set of 18 full-HD videos.Each video has about 1 hour long. The video has a total of 20865 vehicles (cases) which are marked using LiDAR and verified with several referencessuch as GPS tracks. The dataset concerns with the accuracy and the errors of vehicles counting in different situations. The dataset is available for download and contains the videos and metadata for evaluation. Sample image of the dataset is presented in Fig 2.

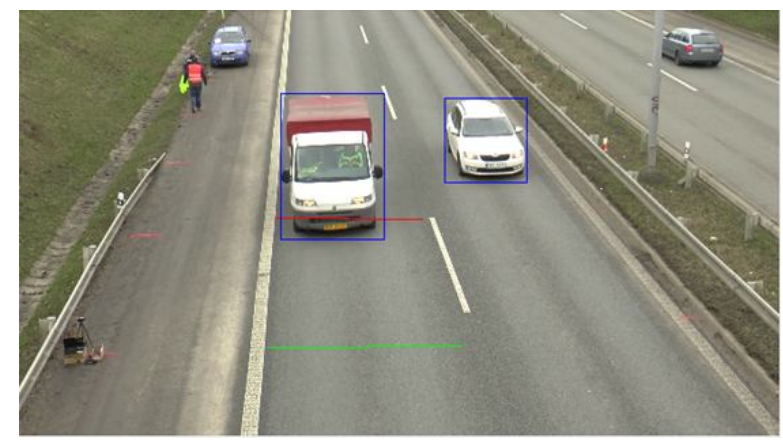

Figure 2: The testing dataset 


\subsection{Automatic Video-basedVehicles Counting (AVVC)}

TheAutomatic Video-based Vehicles Counting (AVVC) model consists of four processing steps which are preprocessing, image segmentation, object identification, vehicle identification, object tracking. The preprocessing includes video pre-processing, image framing, image pre-processing andimage cleaning. The output parametersof vehicle counting are the number of vehicles each lane, the total number of vehicles, critical lane and peak of traffic. Fig 3 below shows an overview of the proposed AVVC model.

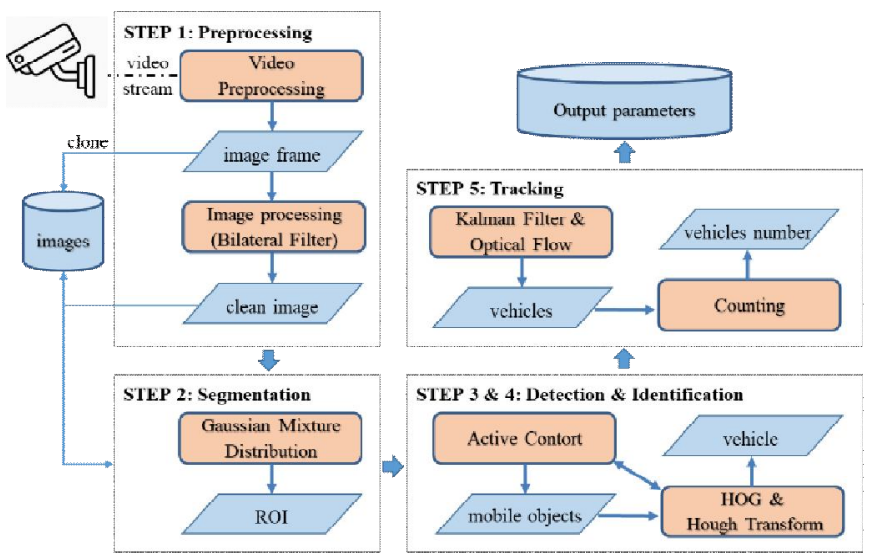

Fig 3. The proposed vehicle counting model

Segmentation is dividing the foreground of an image to a set of segments that collectively cover the entire contents of the image or a set of contours extracted from the image. A traditional method for real-time segmentation of moving object in a video-based system is background subtraction, or computing the error between a background model and processed frames [13].Background subtraction can be generalized as three stages of the process which are pre-processing, background modelling, and foreground detection [14]. The essential demand for background subtraction is to estimate a robust background to deal with the change of illuminate or object. The AVVC model encompasses Gaussian Mixture Distribution to remove the uneven shadow of vehicles, background subtraction then detecting moving objects. It outcomes animproved foreground (without shadow).

For the detection, the model detects the moving object whether it is vehicles or not. The model implements Active Contoursto measure the contours and boundaries of vehicle objects. Contour models describe the object boundaries or any other features of the image to form a parametric curve or contour to count vehicle-based on the number of contours [15], [16]. It has the ability to detect moving object in image frames. Then it measures the size of the object through the length and shape of their contour pixels. The size of the object is compared with standard thresholds to infer the object represents a vehicle or not.The AVVC model integrates Histogram of Oriented Gradient (HOG) and Hough Transform algorithmsto improve the accuracy of vehicle counting and to detect the overlap image and also overhead image.

The tracking process aims to ensure that the counted vehicles do not cause redundant in counting. Each detected vehicle is associated with a Kalman Filter to be tracked until its count is recorded by a counting trigger line lies across the tracking region.A tracked object that has already crossed the predetermined virtual line will be counted as a detected vehicle [17], [18]. The Optical Flow algorithm detects the direction of the vehicle that passes through for better tracking. Subsequently, a rule-based checking is taken into account to correct false countingsuch as car parking or car moving in a backward direction, occlusion and effects caused by errors in the earlier detection stage. The tracked vehicle datais sent to the vehicle counting algorithm for recording the quantity of related vehicle counted parameters.

\subsection{Evaluation Metrics for Vehicle Counting}

The formulas that are used to calculate the accuracy and the error of the vehicles counting as follows [19], [20]:

- Error: in \%. The proportion of the total number of predictionsthat are incorrect.

$$
\operatorname{Error}(\%)=\frac{\mid \text { actual_found-ground_truth } \mid}{\text { actual_found }} \times 100
$$

- Average error:It computes the average number of predictionsthat are incorrect.

$$
\text { Average error }=\frac{\sum \mathfrak{i} \text { error } \mathfrak{i}}{n}
$$

- Accuracy:The proportion of the total number of predictions that are correct.

$$
\text { Accuracy }=100-\text { average error }
$$

- Precision:The standard deviation formula is used to calculate the proportion of the predicted precision.

$$
\text { Precision }=\sqrt{\frac{\sqrt{\sum i(x i-\bar{x})^{2}}}{n-1}}
$$

\section{RESULT AND DISCUSSION}

This section discussing the implementation of the system, the performed tests and the results. A challenge of this work is to select proper thresholdsbecause the camera positioning and angle are changing from one dataset to another. if the threshold is too small or too big, then the system will not detect the vehicles accurately and produce some slack results. Several initial tests are conducted for the setting of the parameters/thresholds of the AVVC model. The nature of trials carried out, including peak periods with various vehicle types, leads to an increase of conclusions between small vehicles (cars) and big vehicles (trucks). A specific method for severe conclusions detection, based on the notion of 
solidity, are carried out and tested.Subsequently, the video threshold is set to 20 , the detection area threshold is set to 1700 and the vehicle size threshold is set to 25000 .

Table 1 shows the data collection of the results in which vehicles counting is performed for lane 1 and lane 2. Ground truth is the actual numberof vehicles. Then the correct counting is presented as 1 and incorrect counting is presented as 0 .

Table 1: Vehicle Counting samples

\begin{tabular}{|l|c|c|c|c|c|}
\hline Dataset & Lane & $\begin{array}{c}\text { Correct } \\
\text { Count }\end{array}$ & Time(S) & $\begin{array}{c}\text { Correct } \\
\text { Count }\end{array}$ & $\begin{array}{c}\text { Ground } \\
\text { Truth }\end{array}$ \\
\hline Video 1 & Lane 1 & 1 & 3.72 & 1 & 1 \\
\cline { 3 - 6 } & & 2 & 4.28 & 0 & 1 \\
\cline { 2 - 6 } & Lane 2 & 1 & 4.68 & 1 & 1 \\
\cline { 3 - 6 } & & 2 & 9.32 & 1 & 1 \\
\hline Video 2 & Lane 1 & 1 & 19.8 & 1 & 1 \\
\cline { 3 - 6 } & & 2 & 20.38 & 1 & 1 \\
\cline { 2 - 6 } & Lane 2 & 1 & 20.2 & 1 & 1 \\
\cline { 3 - 6 } & & 2 & 100.5 & 1 & 1 \\
\hline Video 3 & Lane 1 & 1 & 0.48 & 1 & 1 \\
\cline { 3 - 6 } & & 2 & 0.59 & 1 & 1 \\
\cline { 2 - 6 } & \multirow{2}{*}{ Lane 2 } & 1 & 2.50 & 1 & 0 \\
\cline { 3 - 6 } & & 2 & 2.82 & 1 & 1 \\
\hline
\end{tabular}

Table 2 shows the testing results for each lane and video records. The TFA parametersare vehicles count of the lane, total count of the road, critical lane and peak time.

Table 2: Results of testing parameters

\begin{tabular}{|c|c|c|c|c|c|c|}
\hline \multirow{2}{*}{$\begin{array}{c}\text { TFA } \\
\text { Parameters }\end{array}$} & \multicolumn{2}{|c|}{ Video1 } & \multicolumn{2}{c|}{ Video 2 } & \multicolumn{2}{c|}{ Video 3 } \\
count & $\begin{array}{c}\text { ground } \\
\text { truth }\end{array}$ & $\begin{array}{c}\text { correct } \\
\text { count }\end{array}$ & $\begin{array}{c}\text { ground } \\
\text { truth }\end{array}$ & $\begin{array}{c}\text { correct } \\
\text { count }\end{array}$ & $\begin{array}{c}\text { ground } \\
\text { truth }\end{array}$ \\
\hline $\begin{array}{c}\text { count in } \\
\text { lane 1 }\end{array}$ & 127 & 130 & 19 & 19 & 102 & 109 \\
\hline $\begin{array}{c}\text { count in } \\
\text { lane 2 }\end{array}$ & 55 & 56 & 22 & 23 & 60 & 70 \\
\hline total count & 182 & 186 & 41 & 42 & 162 & 179 \\
\hline critical lane & \multicolumn{2}{|c|}{ Lane 1(right) } & \multicolumn{2}{c|}{ Lane 2(left) } & \multicolumn{2}{c|}{ Lane 1(right) } \\
\hline Peak time & \multicolumn{2}{|c|}{ Lane 1(right) } & \multicolumn{2}{c|}{ Lane 2(left) } & \multicolumn{2}{c|}{ Lane 1(right) } \\
\hline
\end{tabular}

Subsequently, Table 3 shows the accuracy and precision results based on the results of Table 2. This table contains the error results in which video 1 has the lowest error of $3.92 \%$, video 2 has $4.35 \%$ error and video 3 has the highest error of $20.66 \%$. Then, the average error is calculated in which video 1 has $2.05 \%$, video 2 has $2.18 \%$ and video 3 has $10.33 \%$ average error. Video 1 scores the highest accuracy of $97.95 \%$ and video 2 scores the highest precision of $99.07 \%$. The AVVC model achieves average vehicles counting accuracy of 95.14 and precision of $92.81 \%$.
Table 3: The overallresults

\begin{tabular}{|c|c|c|c|c|}
\hline $\begin{array}{c}\text { TFA } \\
\text { Parameters }\end{array}$ & Video 1 & Video 2 & Video 3 & Overall \\
\hline Errors & $3.92 \%$ & $4.35 \%$ & $20.66 \%$ & $9.64 \%$ \\
\hline Average error & $2.05 \%$ & $2.18 \%$ & $10.33 \%$ & $4.85 \%$ \\
\hline Accuracy & $97.95 \%$ & $97.82 \%$ & $89.67 \%$ & 95.14 \\
\hline Precision & $96.12 \%$ & $99.07 \%$ & $83.26 \%$ & 92.81 \\
\hline
\end{tabular}

\section{CONCLUSIONS}

This paper introduced a proposed Automatic Video-based Vehicles Counting (AVVC) for vehiclescounting fromthe video stream. This model extends several parameters of Traffic Flow Analysis (TFA) including vehicles count of the lane, total count of the road, critical lane and peak time.The AVVC model is able to count vehicles more accuratelyand avoid some misleading cases that cause incorrect counting. As a conclusion, the result for the vehicle counting model in this project comprises video 1 that has the lowest error of 3.92\%, video 2 that has an error of $4.35 \%$ and video 3 that has the highest error of $20.66 \%$. Then, the average error of video 1 is $2.05 \%$, video 2 is $2.18 \%$ and video 3 is $10.33 \%$. Video 1 scores the highest accuracy of $97.95 \%$ and video 2 scores the highest precision of $99.07 \%$. The AVVC model achieves average vehicles counting accuracy of 95.14 and precision of $92.81 \%$. Future work considers applying machine learning algorithms to improve the segmentation and counting processes.

\section{ACKNOWLEDGEMENT}

This paper is supported by Research Fund E15501, Research Management Centre, UniversitiTun Hussein Onn Malaysia.

\section{REFERENCES}

1. Z. Al-Ariny, M. A. Abdelwahab, M.Fakhry and E. S. Hasaneen. An Efficient Vehicle Counting Method Using Mask R-CNN. In 2020 International Conference on Innovative Trends in Communication and Computer Engineering (ITCE), IEEE, pp. 232-237, 2020.

2. A. Crouzil, L. Khoudour, P. Valiere and D. N. T. Cong. Automatic vehicle counting system for traffic monitoring. Journal of Electronic Imaging, 25(5), 051207, 2016.

3. K. Adi, A. P. Widodo, C. E. Widodo, A. Pamungkas and A. B. Putranto. Automatic vehicle counting using background subtraction method on gray scale images and morphology operation. In Journal of Physics: Conference Series, IOP Publishing, Vol. 1025, No. 1, p. 012025, 2018.

4. N. Chhadikar, P. Bhamare, K. Patil and S. Kumari. Image processing based Tracking and Counting Vehicles. In 2019 3rd International conference on Electronics, Communication and Aerospace Technology (ICECA), IEEE, pp. 335-339, 2019. 
5. Z. Dai, H. Song, X. Wang, Y. Fang, X. Yun, Z. Zhang and $\mathrm{H}$. Li. Video-based vehicle counting framework. IEEE Access, 7, 64460-64470, 2019.

6. M. Soleh, G. Jati and M. H. Hilman. Multi Object Detection and Tracking Using Optical Flow Density-Hungarian Kalman Filter (Ofd-Hkf) Algorithm for Vehicle Counting. JurnalIImuKomputerdanInformasi, 11(1), 17-26, 2018.

7. D. Kang, Z. Ma and A. B. Chan. Beyond Counting: Comparisons of Density Maps for Crowd Analysis Tasks-Counting, Detection, and Tracking. IEEE Transactions on Circuits and Systems for Video Technology, 29(5), 1408-1422, 2018.

8. C. Wang, A. Musaev, P. Sheinidashtegol and T. Atkison. Towards Detection of Abnormal Vehicle Behavior Using Traffic Cameras. In International Conference on Big Data, Springer Cham, pp. 125-136, 2019.

9. W. Balid, H. Tafish and H. H. Refai. Intelligent vehicle counting and classification sensor for real-time traffic surveillance. IEEE Transactions on Intelligent Transportation Systems, 19(6), 1784-1794, (2017).

10. A. H. Fadel, H. H. Razzaq and S. A. Mostafa. A low complexity partial transmit sequence approach based on hybrid segmentation scheme. Bulletin of Electrical Engineering and Informatics, 9(6), (2020).

11. M. Lei, D. Lefloch, P. Gouton and K. Madani. A video-based real-time vehicle counting system using adaptive background method. In 2018 IEEE International Conference on Signal Image Technology and Internet Based Systems, IEEE, pp. 523-528, 2018.

12. J. Sochor, R. Juránek, J. Španhel, L. Maršık, A. Široký, A. Herout and P. Zemcik. BrnoCompSpeed: Review of traffic camera calibration and comprehensive dataset for monocular speed measurement. arXiv preprint arXiv: 1702.06441, 3(5), 6, (2017).
13. H. Yang and S. Qu. Real-time vehicle detection and counting in complex traffic scenes using background subtraction model with low-rank decomposition. IET Intelligent Transport Systems, 12(1), 75-85, (2017).

14. X. Xiang, M. Zhai, N. Lv and A. El Saddik. Vehicle counting based on vehicle detection and tracking from aerial videos. Sensors, 18(8), 2560, (2018).

15. B. Hardjono, M. G.Rhizma, A. E. Widjaja, H. Tjahyadi and M. J. Josodipuro. Vehicle Counting Evaluation on Low-resolution Images using Software Tools. In Proceedings of the 9th International Conference on Information Communication and Management, pp. 89-94, 2019.

16. Y. Xia, X. Shi, G. Song, Q. Geng, and Y. Liu. Towards improving quality of video-based vehicle counting method for traffic flow estimation. Signal Processing, 120, 672-681.

17. Xu, H., Zhou, W., Zhu, J., Huang, X., \& Wang, W. (2017). Vehicle counting based on double virtual lines. Signal, Image and Video Processing, 11(5), 905-912, (2016).

18. , J. W. Hsieh, S. H. Yu, Y. S. Chen and W. F. Hu. Automatic traffic surveillance system for vehicle tracking and classification. IEEE Transactions on Intelligent Transportation Systems, 7(2), 175-187, 2006.

19. A.ThaeerHammid, O. I.Awad, M. H.Sulaiman, S. S.Gunasekaran,S. A. Mostafa, N.Manoj Kumar, B.A. Khalaf, Y.A. Al-Jawhar, and R.A.Abdulhasan. A Review of Optimization Algorithms in Solving Hydro Generation Scheduling Problems. Energies, 13(11), 2787, (2020).

20. A. B.Annasaheb, V. K.Verma. Data mining classification techniques: A recent survey. International Journal of Emerging Technologies in Engineering Research, 4(8), 51-54, (2016). 